\title{
Editorial: CD1- and MR1-Restricted T Cells in Antimicrobial Immunity
}

\author{
S.M. Mansour Haeryfar ${ }^{1,2,3,4 *}$ and Thierry Mallevaey ${ }^{5}$ \\ ${ }^{1}$ Department of Microbiology and Immunology, Western University, London, ON, Canada, ${ }^{2}$ Division of Clinical Immunology \\ and Allergy, Department of Medicine, Western University, London, ON, Canada, ${ }^{3}$ Centre for Human Immunology, Western \\ University, London, ON, Canada, ${ }^{4}$ Lawson Health Research Institute, London, ON, Canada, ${ }^{5}$ Department of Immunology, \\ University of Toronto, Toronto, ON, Canada
}

Keywords: CD1, MR1, NKT cell, MAIT cell, infection, inflammation, immunity, cytokine

The main function of the immune system is to protect the host against microbial pathogens and their deleterious products. Innate defense mechanisms quickly eliminate infectious intruders or keep them in check until highly specific adaptive responses that also give rise to immunological memory are launched. Major histocompatibility complex (MHC)-restricted T cells are key players of adaptive immunity. The remarkable diversity of their T cell receptors (TCRs) allows for specific recognition of peptides derived from virtually all protein antigens (Ags) including those harbored or even modified by the most vicious pathogens encountered over one's lifetime. Conventional T cells sense and respond to pathogen-derived peptides complexed with polymorphic MHC molecules. This is called the rule of MHC restriction (1).

Recent years have witnessed a growing appreciation for effector and regulatory functions of innate-like $\mathrm{T}$ cells that are restricted by non-polymorphic, MHC-like molecules. These include CD1restricted T cells [e.g., natural killer T (NKT) cells] and MR1-restricted mucosa-associated invariant T (MAIT) cells, which are the subjects of discussion by leading experts in this Research Topic. These "unconventional" $T$ cells may directly target infected cells, but are best known for their ability to swiftly secrete T helper 1 (Th1)-, Th2-, and/or Th17-type cytokines very early in the course of immune responses. These cytokines in turn modulate the function of numerous cell types including NK cells, macrophages, dendritic cells, conventional $\mathrm{CD} 4^{+}$and $\mathrm{CD} 8^{+} \mathrm{T}$ cells and $\mathrm{B}$ cells, all of which play critical roles in innate or adaptive antimicrobial immunity.

CD1 molecules appeared around 300 million years ago. They display a high degree of conservation across vertebrates (2). However, considerable inter-species variation exists in terms of the number of CD1 isoforms expressed. In humans, the CD1 locus encodes five isoforms (i.e., CD1a-e), whereas rodents express only CD1d $(3,4)$. With the sole exception of CD1e, CD1 molecules are cell surface glycoproteins directly participating in lipid Ag presentation to $\mathrm{T}$ cells. CD1a-c, which comprise Group 1 CD1 molecules based on their genomic homology and location, have been a focus of many studies on host responses to Mycobacterium tuberculosis lipids (5). By contrast, certain selflipids and exogenous glycolipids derived from a relatively wide spectrum of pathogens can be presented to NKT cells by CD1d, which is considered the Group 2 CD1 molecule. Positive selection of both type I and type II NKT cells in the thymus also requires their interaction with CD1d (6). These cell types are commonly referred to as invariant NKT ( $i \mathrm{NKT})$ and variant NKT $(v \mathrm{NKT})$ cells, respectively. The discovery of $i \mathrm{NKT}$ cell restriction by CD1d and the ability of these cells to recognize $\alpha$-galactosylceramide ( $\alpha$-GalCer) (7) prompted the invention of $\alpha$-GalCer-loaded CD1d tetramer reagents $(8,9)$. This can be viewed as a technical breakthrough that has allowed for accurate identification and functional analysis of $i$ NKT cells in mice and humans. Subsequently, CD1d tetramers loaded with the myelin-derived glycolipid sulfatide were generated and employed to identify a substantial fraction of $v$ NKT cells (10). Of note, CD1d-restricted, sulfatide-reactive $\gamma \delta$ $\mathrm{T}$ cells have also been described $(11,12)$. To what extent CD1d-restricted $\gamma \delta$ T cells may contribute 
to the resolution of infection is not clearly understood. $\gamma \delta \mathrm{T}$ cells are not a major focus of this Topic, but have been introduced and briefly discussed $(13,14)$.

$i$ NKT cells are perhaps the most widely studied population of CD1-restricted $\mathrm{T}$ cells. They are relatively infrequent in circulation and in most lymphoid and non-lymphoid tissues. However, they amass in the mouse liver and in the human omentum (15), which was dubbed the "abdominal policeman" in 1906 (16). $i$ NKT cells express NK cell markers along with a canonical TCR consisting of an invariant $\alpha$ chain (V $\alpha 14$-J $\alpha 18$ in mice and $\mathrm{V} \alpha 24-\mathrm{J} \alpha 18$ in humans) and one of the only few $\beta$ chain choices, namely mouse $\mathrm{V} \beta 8.2$, V $\beta 2$, or V $\beta 7$ and human V $\beta 11$. Positive selection of $i \mathrm{NKT}$ cells is executed by $\mathrm{CD} 1 \mathrm{~d}^{+} \mathrm{CD} 4^{+} \mathrm{CD}^{+}$thymocytes (6) However, endogenous CD1d ligand(s) involved in $i$ NKT cells' thymic selection have been elusive. KRN7000, an exogenous glycolipid superagonist of $i$ NKT cells, was first extracted from the sea sponge Agelas mauritanius in a screen for novel antitumor compounds (7, 17). It has a unique $\alpha$-GalCer structure and is likely to have originated from microbes forming a symbiotic relationship with $A$. mauritanius. Up until a short time ago, $\alpha$-GalCer was considered unnatural to mammals. However, a recent study documented the presence of $\alpha$-anomeric glycosylceramides including $\alpha$-GalCer, in minute quantities, within mammalian cells, which could serve as endogenous $i \mathrm{NKT}$ cell Ags (18). $\alpha$-GalCer has been utilized as a powerful experimental tool in many mouse studies and as a therapeutic agent in several clinical trials for cancer and viral diseases $(19,20)$.

$i$ NKT cells are among first-line emergency responders to microbes. They quickly accumulate at the sites of infection, injury or inflammation to aid in mobilization and activation of other immune cells (21). When infection alters $i$ NKT cell numbers within a given tissue, it is informative to distinguish between their recruitment into the tissue and their in situ expansion, retention or redistribution. Intravital imaging techniques have permitted the visualization and monitoring of $i$ NKT cells' behavior in live animals $(22,23)$. They have revealed, for instance, that shortly after infection with Borrelia burgdorferi, the causative agent of Lyme disease, hepatic mouse $i$ NKT cells cease to crawl along the liver sinusoids, but instead form clusters and establish firm adhesion with Kupffer cells that have engulfed the bloodborne spirochete (24). By contrast, within the joints, extravascular $i$ NKT cells are not stationary and move along blood vessel walls toward B. burgdorferi (25). This is followed by a direct interaction with the bacterium and its elimination. There currently exists an unmet need to track and quantify CD1d-mediated presentation of pathogen-derived lipids and to investigate the characteristics of the immunological synapses formed between $\mathrm{CD}^{+} \mathrm{d}^{+} \mathrm{Ag}$ presenting cells (APCs) and $i$ NKT cells during infection. As such, antibodies to CD1d:glycolipid complexes and soluble $i$ NKT cell TCR reagents similar to those engineered before $(26,27)$ may prove valuable.

Lipid Ags that can be bound to CD1d and directly detected by $i$ NKT cells are present in a number of pathogenic or commensal bacteria or protozoan parasites $(21,28,29)$. Sphingomonas spp., Bacteroides fragilis, B. burgdorferi, Helicobacter pylori, Streptococcus pneumoniae, Streptococcus agalactiae, Leishmania donovani and Entamoeba histolytica are examples of such microorganisms.
Viruses do not possess lipid ligands for $i$ NKT cell TCRs. However, infection with some viruses (e.g., dengue virus) leads to upregulated CD1d expression consistent with $i$ NKT cell activation while certain others (e.g., herpesviruses and HIV) downregulate CD1d to plausibly evade detection by $i$ NKT cells $(30,31)$. Viral infection may also induce a shift in host cell lipid profiles, thus yielding more "antigenic" CD1d ligands (32). The presence of CD1d:self-lipid complexes is also often required for cytokinemediated stimulation of $i \mathrm{NKT}$ cells during bacterial and viral infections. This typically occurs shortly after microbial components engage APCs' Toll-like receptors and induce interleukin (IL)-12 and IL-18 secretion $(13,33)$. Finally, $i$ NKT cells can be activated in a truly CD1d-independent fashion, for instance by a combination of IL-12 and IL-18 (34) or by group II superantigens of staphylococcal and streptococcal origin $(35,36)$.

Both protective and pathogenic roles for $i \mathrm{NKT}$ cells have been reported in infectious disease models. Perhaps even more intriguing, $i$ NKT cell activation could have seemingly contradictory consequences during infection with different species of the same pathogen (e.g., Chlamydia spp.) (37). CD1d ${ }^{-1-}$ and $J \alpha 18^{-1-}$ mice have been used extensively to address the contributions of $i$ NKT cells in infection and immunity. It is noteworthy that CD1 $\mathrm{d}^{-1-}$ mice are devoid of not only $i$ NKT cells but also $v$ NKT cells. Moreover, Bedel et al. found that the cellular deficiency of the original $\mathrm{J} \alpha 18^{-/-}$mice is more broad than initially thought and that the TCR $\alpha$ repertoire in these mice is shrunk by $\sim 60 \%$ (38). Therefore, except in cases where CD1d $\mathrm{d}^{-1-}$ or J $\alpha 18^{-1-}$ mice have been reconstituted with $i \mathrm{NKT}$ cells as a confirmatory measure, it may be necessary to revisit earlier findings in these animals. This is now possible thanks to the recent development of a new version of $\mathrm{J} \alpha 18^{-1-}$ mice with an exclusive $i \mathrm{NKT}$ cell deficiency (39) and a monoclonal antibody that can selectively deplete $i \mathrm{NKT}$ cells in vivo (40).

The role of $i \mathrm{NKT}$ cells in infection is dictated, at least partially, by the pro- versus anti-inflammatory dominance of the cytokines they produce. For instance, interferon (IFN)- $\gamma$-secreting $i \mathrm{NKT}$ cells contribute to the immunopathology of sepsis in the aftermath of polymicrobial infection, which can be therapeutically attenuated by Th2-skewing glycolipids $(41,42)$. $\alpha$-GalCer analogs with Th1-polarizing properties have also been synthesized and may be considered as adjuvant candidates in preventative vaccination (43) and in immunotherapy of infectious diseases that can be potentially resolved by Th1-dominant responses (44).

$v$ NKT cells, as indicated by their name, have a relatively heterogeneous $\alpha \beta$ TCR repertoire (45). They are present in mice but more prominent in humans $(14,46)$. Unlike $i \mathrm{NKT}$ cells, $v \mathrm{NKT}$ cells are unresponsive to $\alpha$-GalCer. A large sub-population of $v$ NKT cells react with sulfatide, a self-glycolipid that is abundant within the central nervous system, liver, kidney, and pancreas. Several endogenous lipids other than sulfatide have been found to activate $v \mathrm{NKT}$ cells, suggesting that $v \mathrm{NKT}$ cells' recognition mode can be both specific and somewhat promiscuous. Therefore, it is not unreasonable to assume that self-lipids released from infected or damaged cells or even microbial lipids cross-reactive with self-components may be presented by CD1d to elicit $v \mathrm{NKT}$ cell responses. Potent immunomodulatory cytokines secreted by $v \mathrm{NKT}$ cells mediate protection from or pathology associated with 
infection $(14,46)$. They also enable $v$ NKT cells to establish crosstalk with other cell types including but not limited to $i$ NKT cells. In fact, $v$ NKT and $i$ NKT cells may fulfill opposing functions during infection, as exemplified by parasitic infections of mice with Trypanosoma cruzi (47) and Schistosoma mansoni (48). The limited availability of reliable reagents and tools for $v$ NKT cell studies constitutes a hurdle in delineating the significance of these cells in infectious diseases (14). Sulfatide-loaded CD1d tetramers are not sufficiently stable nor do they stain all $v$ NKT cells. The advent of $24 \alpha \beta$ mice, a transgenic mouse line carrying the rearranged V $\alpha 3.2 / \mathrm{V} \beta 9$ TCR of a $v$ NKT cell hybridoma, shed light on certain aspects of $v$ NKT cell biology and development (49). More recently, J $\alpha 18$-deficient, IL-4 reporter (J $\alpha 18^{-/-} 4$ get) mice were employed to characterize $v$ NKT cells at a polyclonal level (50). These mice were found to be responsive to multiple lipid Ags but not to sulfatide and several phospholipids. Therefore, until new models become available, we will continue to depend on these models and on parallel examination of $\mathrm{CD} \mathrm{d}^{-1-}$ and the exclusively $i$ NKT cell-deficient version of $J \alpha 18^{-/-}$mice to study $v \mathrm{NKT}$ cell responses in vivo.

Mucosa-associated invariant $\mathrm{T}$ cells are one of the hottest topics in immunology today $(51,52)$. They express an invariant TCR $\alpha$ chain (mouse V $\alpha 19-J \alpha 33$ and human V $\alpha 7.2-J \alpha 33$ ) (53, 54 ), and undergo positive selection by $\mathrm{MR} 1^{+} \mathrm{CD} 4{ }^{+} \mathrm{CD} 8^{+}$thymocytes (55). Similar to CD1, MR1 is conserved among diverse mammalian species. MAIT cells preferentially home to mucosal tissues - hence their denomination. In human, they circulate at high frequencies in the blood and also make up $\sim 50 \%$ of the entire hepatic $\mathrm{T}$ cell population. MAIT cells are absent from the peripheral tissues of germ-free mice (55), indicating a strict requirement for commensal microflora in MAIT cell homeostasis. It has been hypothesized that gut dysbiosis in diseased states (e.g., type 1 and type 2 diabetes) may change MAIT cell frequencies and functions with metabolic and inflammatory repercussions (29). MAIT cells are rare in wild-type mice, and V $\alpha 19$ transgenic mice were generated to circumvent the feasibility limitations of mouse studies. Although several differences have been reported between mouse and human MAIT cell compartments, recent work suggests that MAIT cells from wild-type mice resemble their human counterparts more closely than previously appreciated (56).

MR1 tetramers loaded with reduced 6-hydroxymethyl-8-Dribityllumazine, a MAIT cell Ag, were recently developed to enable positive identification of mouse and human MAIT cells (57). The vitamin B2 (riboflavin) biosynthesis pathway supplies MAIT cell ligands (58-60). Importantly, this pathway operates in microbes that activate MAIT cells, but not in mammals. However, host-derived metabolites may potentially form adducts with intermediates of the riboflavin pathway to generate MAIT cell

\section{REFERENCES}

1. Zinkernagel RM, Doherty PC. Restriction of in vitro T cell-mediated cytotoxicity in lymphocytic choriomeningitis within a syngeneic or semiallogeneic system. Nature (1974) 248(5450):701-2. doi:10.1038/248701a0

2. Dascher CC. Evolutionary biology of CD1. Curr Top Microbiol Immunol (2007) 314:3-26. neo-antigens (59). MR1 ligands are ubiquitous and harbored by many bacteria, including commensals. Therefore, how in vivo MAIT cell responses are controlled remains to be elucidated. Novel MR1 ligands that do not activate MAIT cells on their own but compete with bacterial and synthetic MAIT cell stimuli have been synthesized (60). This may inspire the development of MAIT cell inhibitors for experimental and therapeutic purposes.

MAIT cells can respond to numerous bacterial strains and yeasts $(51,52)$. These include Klebsiella pneumoniae, Pseudomonas aeruginosa, Lactobacillus acidophilus, Staphylococcus aureus, Staphylococcus epidermidis, Candida glabrata, Candida albicans and Saccharomyces cerevisiae. To test the in vivo significance of MAIT cells in anti-bacterial immunity, $\mathrm{MR}^{-/-}$mice have been utilized and shown to be unable to control infection with $\mathrm{K}$. pneumoniae, Mycobacterium bovis bacillus Calmette-Guérin (BCG) or Francisella tularensis (61-63). Last but not the least, MAIT cells can be activated by a combination of IL-12 and IL-18 in an MR1independent manner (64), which may be important for antiviral defense.

Innate-like $\mathrm{T}$ cells are fast-acting and occupy strategic locations in the body. Unlike classical MHC molecules, CD1 and MR1 exhibit limited polymorphism. Therefore, it is only fitting that CD1 and MR1 ligands are considered by many as attractive targets for vaccination of genetically diverse human populations. Despite gaps in our knowledge in this exciting area, which are outlined by experts in this Topic, the availability of powerful tools, reagents and models has fueled further interest in CD1and MR1-restricted T lymphocytes. $\alpha$-GalCer has been used in clinical trials, and Th1- and Th2-promoting, disease/infectiontailored glycolipid agonists of $i \mathrm{NKT}$ cells may find their way into clinical practice in the future. Furthermore, it is not too farfetched to anticipate that once the role of MAIT cells in various infectious diseases is known, their manipulation by synthetic ligands and inhibitors can be achieved and potentially used in immunotherapeutic protocols. The time is ripe for both curiositydriven and translational studies on CD1- and MR1-restricted T cells.

\section{AUTHOR CONTRIBUTIONS}

SMMH and TM served as co-editors for this Research Topic. SMMH wrote the Editorial.

\section{ACKNOWLEDGMENTS}

We are grateful to our colleagues, both the contributors and the reviewers, for their expert participation in this Research Topic. We apologize to investigators whose work was not cited due to space constraints.

3. Calabi F, Jarvis JM, Martin L, Milstein C. Two classes of CD1 genes. Eur J Immunol (1989) 19(2):285-92. doi:10.1002/eji.1830190211

4. Adams EJ. Lipid presentation by human CD1 molecules and the diverse T cell populations that respond to them. Curr Opin Immunol (2014) 26:1-6. doi:10.1016/j.coi.2013.09.005

5. Siddiqui S, Visvabharathy L, Wang CR. Role of group 1 CD1-restricted T cells in infectious disease. Front Immunol (2015) 6:337. doi:10.3389/fimmu.2015.00337 
6. Bendelac A. Positive selection of mouse NK1+ T cells by CD1-expressing cortical thymocytes. J Exp Med (1995) 182(6):2091-6. doi:10.1084/jem.182.6.2091

7. Kawano T, Cui J, Koezuka Y, Toura I, Kaneko Y, Motoki K, et al. CD1drestricted and TCR-mediated activation of valpha14 NKT cells by glycosylceramides. Science (1997) 278(5343):1626-9. doi:10.1126/science.278.5343.1626

8. Benlagha K, Weiss A, Beavis A, Teyton L, Bendelac A. In vivo identification of glycolipid antigen-specific T cells using fluorescent CD1d tetramers. J Exp Med (2000) 191(11):1895-903. doi:10.1084/jem.191.11.1895

9. Matsuda JL, Naidenko OV, Gapin L, Nakayama T, Taniguchi M, Wang CR, et al. Tracking the response of natural killer T cells to a glycolipid antigen using CD1d tetramers. J Exp Med (2000) 192(5):741-54. doi:10.1084/jem.192.5.741

10. Jahng A, Maricic I, Aguilera C, Cardell S, Halder RC, Kumar V. Prevention of autoimmunity by targeting a distinct, noninvariant CD1d-reactive T cell population reactive to sulfatide. J Exp Med (2004) 199(7):947-57. doi:10.1084/ jem.20031389

11. Bai L, Picard D, Anderson B, Chaudhary V, Luoma A, Jabri B, et al. The majority of CD1d-sulfatide-specific T cells in human blood use a semiinvariant Vdelta1 TCR. Eur J Immunol (2012) 42(9):2505-10. doi:10.1002/eji.201242531

12. Luoma AM, Castro CD, Mayassi T, Bembinster LA, Bai L, Picard D, et al. Crystal structure of Vdelta1 $\mathrm{T}$ cell receptor in complex with CD1d-sulfatide shows MHC-like recognition of a self-lipid by human gammadelta T cells. Immunity (2013) 39(6):1032-42. doi:10.1016/j.immuni.2013.11.001

13. Gao Y, Williams AP. Role of innate T Cells in anti-bacterial immunity. Front Immunol (2015) 6:302. doi:10.3389/fimmu.2015.00302

14. Macho-Fernandez E, Brigl M. The extended family of CD1d-restricted NKT cells: sifting through a mixed bag of TCRs, antigens, and functions. Front Immunol (2015) 6:362. doi:10.3389/fimmu.2015.00362

15. Lynch L, O'Shea D, Winter DC, Geoghegan J, Doherty DG, O'Farrelly C. Invariant NKT cells and CD1d(+) cells amass in human omentum and are depleted in patients with cancer and obesity. Eur J Immunol (2009) 39(7):1893-901. doi:10.1002/eji.200939349

16. Morison R. Remarks on some functions of the omentum. Br Med J (1906) 1(2350):76-8. doi:10.1136/bmj.1.2350.76

17. Morita M, Motoki K, Akimoto K, Natori T, Sakai T, Sawa E, et al. Structureactivity relationship of alpha-galactosylceramides against B16-bearing mice. $J$ Med Chem (1995) 38(12):2176-87. doi:10.1021/jm00012a018

18. Kain L, Webb B, Anderson BL, Deng S, Holt M, Costanzo A, et al. The identification of the endogenous ligands of natural killer $\mathrm{T}$ cells reveals the presence of mammalian alpha-linked glycosylceramides. Immunity (2014) 41(4):543-54. doi:10.1016/j.immuni.2014.08.017

19. van den Heuvel MJ, Garg N, Van Kaer L, Haeryfar SM. NKT cell costimulation: experimental progress and therapeutic promise. Trends Mol Med (2011) 17(2):65-77. doi:10.1016/j.molmed.2010.10.007

20. Guo T, Chamoto K, Hirano N. Adoptive T cell therapy targeting CD1 and MR1. Front Immunol (2015) 6:247. doi:10.3389/fimmu.2015.00247

21. Slauenwhite D, Johnston B. Regulation of NKT cell localization in homeostasis and infection. Front Immunol (2015) 6:255. doi:10.3389/fimmu.2015.00255

22. Liew PX, Kubes P. Intravital imaging - dynamic insights into natural killer $\mathrm{T}$ cell biology. Front Immunol (2015) 6:240. doi:10.3389/fimmu.2015.00240

23. Delovitch TL. Imaging of NKT cell recirculation and tissue migration during antimicrobial immunity. Front Immunol (2015) 6:356. doi:10.3389/fimmu. 2015.00356

24. Lee WY, Moriarty TJ, Wong CH, Zhou H, Strieter RM, van Rooijen N, et al. An intravascular immune response to Borrelia burgdorferi involves Kupffer cells and iNKT cells. Nat Immunol (2010) 11(4):295-302. doi:10.1038/ni.1855

25. Lee WY, Sanz MJ, Wong CH, Hardy PO, Salman-Dilgimen A, Moriarty TJ, et al. Invariant natural killer T cells act as an extravascular cytotoxic barrier for jointinvading Lyme Borrelia. Proc Natl Acad Sci U S A (2014) 111(38):13936-41. doi:10.1073/pnas.1404769111

26. McCarthy C, Shepherd D, Fleire S, Stronge VS, Koch M, Illarionov PA, et al. The length of lipids bound to human CD1d molecules modulates the affinity of NKT cell TCR and the threshold of NKT cell activation. J Exp Med (2007) 204(5):1131-44. doi:10.1084/jem.20062342

27. Im JS, Arora P, Bricard G, Molano A, Venkataswamy MM, Baine I, et al. Kinetics and cellular site of glycolipid loading control the outcome of natural killer $\mathrm{T}$ cell activation. Immunity (2009) 30(6):888-98. doi:10.1016/j.immuni.2009. 03.022

28. Zajonc DM, Girardi E. Recognition of microbial glycolipids by natural killer T cells. Front Immunol (2015) 6:400. doi:10.3389/fimmu.2015.00400
29. Magalhaes I, Kiaf B, Lehuen A. iNKT and MAIT cell alterations in diabetes. Front Immunol (2015) 6:341. doi:10.3389/fimmu.2015.00341

30. Chung BK, Priatel JJ, Tan R. CD1d expression and invariant NKT cell responses in herpesvirus infections. Front Immunol (2015) 6:312. doi:10.3389/fimmu. 2015.00312

31. Opasawatchai A, Matangkasombut P. iNKT cells and their potential lipid ligands during viral infection. Front Immunol (2015) 6:378. doi:10.3389/fimmu. 2015.00378

32. Zeissig S, Murata K, Sweet L, Publicover J, Hu Z, Kaser A, et al. Hepatitis B virusinduced lipid alterations contribute to natural killer $\mathrm{T}$ cell-dependent protective immunity. Nat Med (2012) 18(7):1060-8. doi:10.1038/nm.2811

33. Van Kaer L, Parekh VV, Wu L. The response of CD1d-restricted invariant NKT cells to microbial pathogens and their products. Front Immunol (2015) 6:226. doi:10.3389/fimmu.2015.00226

34. Leite-De-Moraes MC, Hameg A, Arnould A, Machavoine F, Koezuka Y, Schneider E, et al. A distinct IL-18-induced pathway to fully activate NK T lymphocytes independently from TCR engagement. J Immunol (1999) 163(11):5871-6.

35. Hayworth JL, Mazzuca DM, Maleki Vareki S, Welch I, McCormick JK, Haeryfar SM. CD1d-independent activation of mouse and human iNKT cells by bacterial superantigens. Immunol Cell Biol (2012) 90(7):699-709. doi:10.1038/icb. 2011.90

36. Rieder SA, Nagarkatti P, Nagarkatti M. CD1d-independent activation of invariant natural killer $\mathrm{T}$ cells by staphylococcal enterotoxin $\mathrm{B}$ through major histocompatibility complex class II/T cell receptor interaction results in acute lung injury. Infect Immun (2011) 79(8):3141-8. doi:10.1128/IAI.00177-11

37. Shekhar S, Joyee AG, Yang X. Dynamics of NKT-cell responses to chlamydial infection. Front Immunol (2015) 6:233. doi:10.3389/fimmu.2015.00233

38. Bedel R, Matsuda JL, Brigl M, White J, Kappler J, Marrack P, et al. Lower TCR repertoire diversity in Traj18-deficient mice. Nat Immunol (2012) 13(8):705-6. doi:10.1038/ni.2347

39. Chandra S, Zhao M, Budelsky A, de Mingo Pulido A, Day J, Fu Z, et al. A new mouse strain for the analysis of invariant NKT cell function. Nat Immunol (2015) 16(8):799-800. doi:10.1038/ni.3203

40. Scheuplein F, Lamont DJ, Poynter ME, Boyson JE, Serreze D, Lundblad LK, et al. Mouse invariant monoclonal antibody NKT14: a novel tool to manipulate iNKT cell function in vivo. PLoS One (2015) 10(10):e0140729. doi:10.1371/journal. pone.0140729

41. Anantha RV, Mazzuca DM, Xu SX, Porcelli SA, Fraser DD, Martin CM, et al. T helper type 2-polarized invariant natural killer $\mathrm{T}$ cells reduce disease severity in acute intra-abdominal sepsis. Clin Exp Immunol (2014) 178(2):292-309. doi:10.1111/cei.12404

42. Szabo PA, Anantha RV, Shaler CR, McCormick JK, Haeryfar SM. CD1d- and MR1-restricted T cells in sepsis. Front Immunol (2015) 6:401. doi:10.3389/ fimmu.2015.00401

43. Kopecky-Bromberg SA, Fraser KA, Pica N, Carnero E, Moran TM, Franck RW, et al. Alpha-C-galactosylceramide as an adjuvant for a live attenuated influenza virus vaccine. Vaccine (2009) 27(28):3766-74. doi:10.1016/j.vaccine. 2009.03.090

44. Schmieg J, Yang G, Franck RW, Tsuji M. Superior protection against malaria and melanoma metastases by a C-glycoside analogue of the natural killer T cell ligand alpha-galactosylceramide. J Exp Med (2003) 198(11):1631-41. doi:10. 1084/jem.20031192

45. Cardell S, Tangri S, Chan S, Kronenberg M, Benoist C, Mathis D. CD1-restricted CD4+ T cells in major histocompatibility complex class II-deficient mice. J Exp Med (1995) 182(4):993-1004. doi:10.1084/jem.182.4.993

46. Marrero I, Ware R, Kumar V. Type II NKT cells in inflammation, autoimmunity, microbial immunity, and cancer. Front Immunol (2015) 6:316. doi:10.3389/ fimmu.2015.00316

47. Duthie MS, Kahn M, White M, Kapur RP, Kahn SJ. Critical proinflammatory and anti-inflammatory functions of different subsets of CD1d-restricted natural killer T cells during Trypanosoma cruzi infection. Infect Immun (2005) 73(1):181-92. doi:10.1128/IAI.73.1.181-192.2005

48. Mallevaey T, Fontaine J, Breuilh L, Paget C, Castro-Keller A, Vendeville C, et al. Invariant and noninvariant natural killer $\mathrm{T}$ cells exert opposite regulatory functions on the immune response during murine schistosomiasis. Infect Immun (2007) 75(5):2171-80. doi:10.1128/IAI.01178-06

49. Skold M, Faizunnessa NN, Wang CR, Cardell S. CD1d-specific NK1.1+ T cells with a transgenic variant TCR. J Immunol (2000) 165(1):168-74. doi:10.4049/ jimmunol.165.1.168 
50. Zhao J, Weng X, Bagchi S, Wang CR. Polyclonal type II natural killer T cells require PLZF and SAP for their development and contribute to CpG-mediated antitumor response. Proc Natl Acad Sci U S A (2014) 111(7):2674-9. doi:10. 1073/pnas.1323845111

51. Howson LJ, Salio M, Cerundolo V. MR1-restricted mucosal-associated invariant $\mathrm{T}$ cells and their activation during infectious diseases. Front Immunol (2015) 6:303. doi:10.3389/fimmu.2015.00303

52. Napier RJ, Adams EJ, Gold MC, Lewinsohn DM. The role of mucosal associated invariant T cells in antimicrobial immunity. Front Immunol (2015) 6:344. doi: 10.3389/fimmu.2015.00344

53. Porcelli S, Yockey CE, Brenner MB, Balk SP. Analysis of T cell antigen receptor (TCR) expression by human peripheral blood CD4-8- alpha/beta T cells demonstrates preferential use of several $\mathrm{V}$ beta genes and an invariant TCR alpha chain. J Exp Med (1993) 178(1):1-16. doi:10.1084/jem.178.1.1

54. Tilloy F, Treiner E, Park SH, Garcia C, Lemonnier F, de la Salle H, et al. An invariant $\mathrm{T}$ cell receptor alpha chain defines a novel TAP-independent major histocompatibility complex class Ib-restricted alpha/beta T cell subpopulation in mammals. J Exp Med (1999) 189(12):1907-21. doi:10.1084/jem.189.12.1907

55. Treiner E, Duban L, Bahram S, Radosavljevic M, Wanner V, Tilloy F, et al. Selection of evolutionarily conserved mucosal-associated invariant $\mathrm{T}$ cells by MR1. Nature (2003) 422(6928):164-9. doi:10.1038/nature01433

56. Rahimpour A, Koay HF, Enders A, Clanchy R, Eckle SB, Meehan B, et al. Identification of phenotypically and functionally heterogeneous mouse mucosal-associated invariant T cells using MR1 tetramers. J Exp Med (2015) 212(7):1095-108. doi:10.1084/jem.20142110

57. Reantragoon R, Corbett AJ, Sakala IG, Gherardin NA, Furness JB, Chen Z, et al. Antigen-loaded MR1 tetramers define T cell receptor heterogeneity in mucosalassociated invariant T cells. J Exp Med (2013) 210(11):2305-20. doi:10.1084/ jem.20130958

58. Kjer-Nielsen L, Patel O, Corbett AJ, Le Nours J, Meehan B, Liu L, et al. MR1 presents microbial vitamin B metabolites to MAIT cells. Nature (2012) 491(7426):717-23. doi:10.1038/nature 11605
59. Corbett AJ, Eckle SB, Birkinshaw RW, Liu L, Patel O, Mahony J, et al. T-cell activation by transitory neo-antigens derived from distinct microbial pathways. Nature (2014) 509(7500):361-5. doi:10.1038/nature13160

60. Soudais C, Samassa F, Sarkis M, Le Bourhis L, Bessoles S, Blanot D, et al. In vitro and in vivo analysis of the gram-negative bacteria-derived riboflavin precursor derivatives activating mouse MAIT cells. J Immunol (2015) 194(10):4641-9. doi:10.4049/jimmunol.1403224

61. Georgel P, Radosavljevic M, Macquin C, Bahram S. The non-conventional MHC class I MR1 molecule controls infection by Klebsiella pneumoniae in mice. Mol Immunol (2011) 48(5):769-75. doi:10.1016/j.molimm.2010.12.002

62. Chua WJ, Truscott SM, Eickhoff CS, Blazevic A, Hoft DF, Hansen TH. Polyclonal mucosa-associated invariant $\mathrm{T}$ cells have unique innate functions in bacterial infection. Infect Immun (2012) 80(9):3256-67. doi:10.1128/IAI.00279-12

63. Meierovics A, Yankelevich WJ, Cowley SC. MAIT cells are critical for optimal mucosal immune responses during in vivo pulmonary bacterial infection. Proc Natl Acad Sci U S A (2013) 110(33):E3119-28. doi:10.1073/pnas.1302799110

64. Ussher JE, Bilton M, Attwod E, Shadwell J, Richardson R, de Lara C, et al. CD161++ CD8+ T cells, including the MAIT cell subset, are specifically activated by IL-12+IL-18 in a TCR-independent manner. Eur J Immunol (2014) 44(1):195-203. doi:10.1002/eji.201343509

Conflict of Interest Statement: The authors declare that the research was conducted in the absence of any commercial or financial relationships that could be construed as a potential conflict of interest.

Copyright (C) 2015 Haeryfar and Mallevaey. This is an open-access article distributed under the terms of the Creative Commons Attribution License (CC BY). The use, distribution or reproduction in other forums is permitted, provided the original author(s) or licensor are credited and that the original publication in this journal is cited, in accordance with accepted academic practice. No use, distribution or reproduction is permitted which does not comply with these terms. 